Chapman University

Chapman University Digital Commons

8-2-2020

\title{
A Pilot Study of the Preliminary Efficacy of Pain Buddy: A Novel Intervention for the Management of Children's Cancer-Related Pain
}

John F. Hunter

Amanda M. Acevedo

Sergio Gago-Masague

Alexandra Kain

Christine Yun

See next page for additional authors

Follow this and additional works at: https://digitalcommons.chapman.edu/psychology_articles

Part of the Child Psychology Commons, Oncology Commons, Pain Management Commons, and the Pediatrics Commons 


\section{A Pilot Study of the Preliminary Efficacy of Pain Buddy: A Novel Intervention for the Management of Children's Cancer-Related Pain}

\section{Comments}

This is the accepted version of the following article:

Hunter JF, Acevedo AM, Gago-Masague S, et al. A pilot study of the preliminary efficacy of Pain Buddy: a novel intervention for the management of children's cancer-related pain. Pediatr Blood Cancer. 2020; 67:e28278.

which has been published in final form at https://doi.org/10.1002/pbc.28278. This article may be used for non-commercial purposes in accordance with Wiley Terms and Conditions for Self-Archiving.

\section{Copyright}

Wiley

\section{Authors}

John F. Hunter, Amanda M. Acevedo, Sergio Gago-Masague, Alexandra Kain, Christine Yun, Lilibeth Torno, Brooke N. Jenkins, and Michelle A. Fortier 


\title{
A Pilot Study of the Preliminary Efficacy of Pain Buddy: A Novel Intervention for the Management of Children's Cancer-Related Pain
}

Pediatric Blood \& Cancer

\author{
John F. Hunter ${ }^{1}$ \\ Amanda M. Acevedo ${ }^{1}$ \\ Sergio Gago-Masague ${ }^{2}$ \\ Alexandra Kain ${ }^{3}$ \\ Christine Yun $^{4}$ \\ Lilibeth Torno ${ }^{4,5}$ \\ Brooke N. Jenkins ${ }^{3,6,7}$ \\ Michelle A. Fortier $3,6,8,9$
}

${ }^{1}$ Department of Psychological Science, University of California-Irvine, Irvine, CA, USA

${ }^{2}$ Department of Computer Science, University of California-Irvine, Irvine, CA, USA

${ }^{3}$ Center on Stress \& Health, School of Medicine, University of California-Irvine, Orange, CA, USA

${ }^{4}$ CHOC Children's Hospital, Hyundai Cancer Institute, Orange, CA, USA

${ }^{5}$ Department of Pediatrics, School of Medicine, University of California-Irvine, Irvine, CA, USA

${ }^{6}$ Department of Anesthesiology and Perioperative Care, University of California-Irvine, Irvine, CA, USA

${ }^{7}$ Department of Psychology, Chapman University, Orange, CA, USA

${ }^{8}$ Sue \& Bill Gross School of Nursing, University of California-Irvine, Irvine, CA, USA

${ }^{9}$ Department of Pediatric Psychology, Children's Hospital of Orange County, Orange, CA, USA

Corresponding author details

Dr. M.A. Fortier, UCI Center on Stress \& Health, School of Medicine, University of CaliforniaIrvine, Orange, CA, 92868, USA.

Email: mfortier@uci.edu

Abstract word count $=223$

Main text word count $=3,007$

1 Table

2 Figures

1 Supplemental File

Running title: Preliminary efficacy of Pain Buddy

Keywords: Pain Buddy, mHealth, pediatric oncology, pain management 


\begin{abstract}
Objectives: Cancer-related pain in children is prevalent and undermanaged. Mobile health (mHealth) applications provide a promising avenue to address the gap in pain management in children with cancer. Pain Buddy is a multi-component mHealth application developed to manage cancer-related pain in children. The goal of this paper is to present preliminary efficacy data of the impact of Pain Buddy on children's pain severity and frequency. Methods: In a randomized controlled trial over 60 days, children $(N=48)$ reported daily pain on a tablet while receiving usual care. Those in the intervention group $(N=20)$ received remote symptom monitoring and skills training for pain management. Children in the attention control group $(N=28)$ only reported on their pain. Results: Both groups experienced significant reductions in average daily pain over the study period $(B=-0.10, z=-3.40, p=.001)$, with no group differences evident $(z=-0.83, p=.40)$. However, the intervention group reported significantly fewer instances of moderate to severe pain compared to the control group, $t(4125)=2.67, p=0.007$. In addition, the intervention group reported no instances of moderate to severe pain toward the end of the study period. Conclusion: Pain Buddy is an innovative and interactive mHealth application that aims to improve pain and symptom management among children with cancer. The findings from this pilot study suggest that Pain Buddy may aid in the reduction of pain severity in children during cancer treatment.
\end{abstract}




\section{INTRODUCTION}

Over 10,000 children in the United States are diagnosed with cancer every year ${ }^{1}$ and significant symptoms, such as recurrent pain, often accompany its treatment ${ }^{2}$. Furthermore, children are in particular need of easily implementable interventions because the majority of pediatric pain management responsibility has shifted from medical settings to home environments $^{3}$. Because mobile health (mHealth) applications have great potential to improve pediatric pain management ${ }^{4}$, our group developed Pain Buddy as an intervention designed to reduce pain during cancer treatment by facilitating patient-provider symptom communication, providing children training in cognitive and behavioral pain management strategies, and delivering these techniques in an effective and engaging manner ${ }^{5}$. Users of the present version of Pain Buddy are asked to report on their daily pain and symptoms via a computer tablet and that information is provided in real time to clinicians in order to promptly address any symptoms that warrant intervention. Thus, Pain Buddy bridges the research-to-practice gap in the current state of care for children undergoing cancer treatment by providing a medium by which pain and symptoms can be better managed in the home setting.

Retrospective pain reporting can be inaccurate and thus hinder the effectiveness of pain management strategies in the medical setting ${ }^{6}$; however, tracking symptoms in real time through e-diaries is a successful strategy that augments patient-provider communication ${ }^{5-7}$. Accordingly, Pain Buddy collects twice daily e-diary symptom reports (e.g., pain, nausea, fatigue) and provides a remote symptom monitoring communication system that allows healthcare providers to track patterns of symptoms and provide timely intervention to patients in need. The data from Pain Buddy are routed through a server and can be accessed by the appropriate healthcare provider through an administrative portal via the Pain Buddy web app. When a child reports 
symptoms that warrant intervention (determined based upon an algorithm developed in collaboration with the treatment team), the specific symptoms reported are sent immediately to the appropriate provider via text or email message. In addition, in order to address the researchto-practice gap that has limited the translation of evidence-based strategies into the home setting $^{8}$, Pain Buddy incorporates coping skills shown to be effective in the management of pain, such as diaphragmatic breathing ${ }^{9}$, progressive muscle relaxation ${ }^{10}$, guided imagery ${ }^{11}$, distraction $^{12}$, and mindfulness. Trainings for these skills can be accessed at any time and are also linked to children's symptom reports such that based upon symptom severity, duration, frequency, and/or distress, children are directed to the skills component of Pain Buddy. To overcome problems with lack of engagement that have limited the success of similar applications ${ }^{13}$, Pain Buddy includes features such as a virtual store in which children can use coins accumulated during use of the skills training exercises to customize their personalized avatar and purchase additional background themes.

There are several other mHealth interventions targeting pediatric pain such as WebMap ${ }^{14}$ and Pain Squad $+{ }^{15}$ that have been developed and evaluated. Pain Squad + is an innovative app that addresses pediatric pain and allows for real-time communication with healthcare providers. WebMap is an online platform that provides evidence-based pain management skills training for pediatric pain. However, Pain Buddy is unique because it combines all of these elements and focuses specifically on cancer-related pain and symptoms, contains real-time communication features with healthcare providers, and has extensive pain management skills training instruction, including strategies such as mindfulness, imagery, and distraction that can be practiced over time to develop competency. We have previously reported on the development and usability/feasibility testing of Pain Buddy ${ }^{5}$. The purpose of this paper is to present preliminary 
findings from a pilot study examining the efficacy of Pain Buddy for reducing reports of average pain and instances of moderate to severe pain using a randomized controlled trial over a period of 60 days. We hypothesized that individuals in the intervention group would report greater reductions of average daily pain and fewer instances of moderate to severe pain than those in the control group.

\section{METHOD}

\section{Design \& Participants}

A prospective, observational cohort study was conducted to investigate the efficacy of Pain Buddy. This trial was approved by the Institutional Review Boards at Children's Hospital of Orange County (CHOC Children's) and the University of California, Irvine and registered with ClinicalTrials.gov (NCT03384134). Figure 1 presents a CONSORT flow diagram of participant recruitment. Children were eligible to participate if they were between the ages of 8-18 years, currently undergoing cancer treatment at a major children's hospital, have home internet access, and were able to speak, read and write in English. Participants were excluded if they had a cognitive or developmental delay that would prevent them from being able to use Pain Buddy. A total of 447 children were assessed for eligibility and 48 children completed the study (see Table 1 for demographics). Our sample size was determined based on published recommendations for pilot studies ${ }^{16}$ and expected effect sizes from similar studies ${ }^{15}$. The majority of participants (71\%) were diagnosed with Acute Lymphoblastic Leukemia.

\section{Procedures}

Parents and children were approached in outpatient clinics, assessed for eligibility and consented to participate. Parent consent forms and questionnaires were available in English or Spanish. After undergoing the consent and training processes, all children completed baseline 
assessments and were randomly assigned to the intervention $(n=20)$ or attention control group $(n=28)$. Randomization was stratified by age (8-10, 11-14, and 15-18) and then time since diagnosis ( $<6$ months, 6-12 months, +1 year) by eight project researchers using an online randomization generator. Participants in both conditions were provided with the Pain Buddy application on a study-specific Android tablet and instructed to complete twice daily symptom diaries (once in the morning, once in the evening) in English using the Memorial Symptom Assessment Scale (MSAS) ${ }^{17}$ and Adolescent Pediatric Pain Tool (APPT) ${ }^{18}$. The MSAS evaluated the frequency, severity, and distress related to particular symptoms since the time of their last diary entry. If pain was endorsed on the MSAS, children filled out the APPT to report their pain intensity. Both groups were instructed to complete these surveys each day, however, only the intervention group had access to the skills training and remote symptom monitoring components.

For children in the intervention group, an algorithm was used to determine if their pain ratings met a certain threshold that warranted intervention, which included notification of a nurse practitioner (NP) via the remote symptom monitoring component. The algorithm triggered notification if a pain rating was over 7.5 , or if there was a combination of reporting significant frequency, severity, and distress of the pain ( $\operatorname{see}^{5}$ for full description of intervention algorithm). Symptom alerts were immediately sent to the NP, who is a member of the oncology treatment team and was the only NP involved in this study. The alerts were treated as a "sick call," wherein a family contacts the clinic when a child is experiencing new or worsening symptoms. The response to the pain alert was not dictated by the study protocol. That is, the NP handled the pain alert as she would if a "sick call" had been made. Thus, Pain Buddy does not necessarily introduce a change in medical treatment but allows for increased and more immediate awareness 
of pain data that allowed for more prompt intervention. Children used the Pain Buddy program for 60 days in addition to receiving usual care.

\section{Measures}

\section{Adolescent Pediatric Pain Tool (APPT)}

The APPT is a multidimensional, valid and reliable pain instrument. If pain was endorsed on the MSAS during a daily diary entry, then children were presented with a body outline via the APPT and asked to select the areas where they felt pain since their last diary entry. Following this step, self-reported pain intensity was reported by the child on a visual analog scale from 0 100 in which the child was asked, "How much pain did you have, on average, since your last diary entry". This type of visual scale has been validated for children 8-18 years of age (Stinson, Kavanagh, Yamada, Gill, \& Stevens, 2006). Our primary outcome of average daily pain was derived from the APPT. The secondary outcome considered was instances of moderate to severe pain, defined as pain episodes greater than or equal to 50 .

\section{Memorial Symptom Assessment Scale (MSAS)}

This instrument evaluated the presence, frequency and effect of particular symptoms since the time of the last diary entry. Children were presented with a symptom description and asked to select "yes" or "no" if they have experienced it. If the child responded "yes", then follow-up questions asked the child to describe the frequency of its occurrence, the severity of its impact, and the distress it caused. Children also had the option to list additional symptoms and associated effects. This scale has two different versions that are designed for specific age groups. The MSAS 7-12 assesses 8 symptoms and is designed for children 7-12 years old (Collins et al., 2000). The MSAS 10-18 assesses 30 symptoms and is designed for children 10-18 years old. For 
this paper, the MSAS was simply used as a tool to determine if it was necessary for the child to complete the APPT during that particular diary entry.

\section{Participant Characteristics}

Parents reported via a demographic survey on child age, sex, race/ethnicity, primary language spoken at home, and family income. Diagnosis and time since diagnosis were abstracted from the medical record.

\section{Approach to Data Analysis}

Preliminary analyses were conducted to examine group differences on sociodemographic variables of interest. Independent samples t-tests were used to compare differences in continuous variables (age, time since diagnosis), a Mann-Whitney U test was used to compare differences in skewed continuous variables (income), and chi-squared analyses were used to compare differences in categorical variables (sex, race/ethnicity, primary language spoken at home, diagnosis). Pain severity from the two e-diary pain reports from each day were averaged together into an average daily pain severity score. Linear growth curve modeling was used to investigate the trajectory of pain reports over the course of the study for both groups. Maximum likelihood estimation was used to handle missing data for growth curve modeling analyses and listwise deletion was used for all other analyses. Average daily pain scores were checked for skewness and kurtosis, with results showing left-skewness. Therefore, a Mann-Whitney-Wilcoxon sum rank test was used to test for differences between the intervention and control groups on average daily pain severity. Frequency of moderate to severe pain episodes were compared between groups using an independent samples t-test.

\section{RESULTS}


Across both groups, children completed $61.2 \%$ of the daily diary entries. Within the Pain Buddy group, on average children used 7 skills trainings for a total of 15 minutes over the course of the study. Unconditional linear growth curve analyses indicated that reports of average daily pain significantly decreased over the course of the study, $B=-0.10, z=-3.40, p=0.001,95 \%$ CI $[-$ $.1594,-.0429]$. Mann-Whitney-Wilcoxon tests indicated that the reports of average daily pain over the course of the study for the Pain Buddy group were not significantly different than the reports of average daily pain for the attention control group, $z=-0.83, p=0.40$ (see Figure 2). However, children in the intervention group reported significantly fewer instances of moderate to severe pain compared to the control group, $t(2145)=2.67, p=0.008,95 \%$ CI $[.0036, .0235]$. Specifically, children in the Pain Buddy condition experienced 6 moderate to severe pain episodes compared to 24 moderate to severe pain episodes experienced by the attention control group. Furthermore, children in the intervention condition only reported moderate to severe pain episodes during the first 18 days of the study, whereas the control group reported these episodes through Day 53.

\section{DISCUSSION}

Under the conditions of this study, we found that children undergoing treatment for cancer experienced a decrease in pain severity over time, which did not differ between the intervention and attention control groups. The overall decrease in pain severity for both groups may have simply been due to the time elapsed since diagnosis/treatment. Findings also showed that children in the active intervention condition of Pain Buddy, that is, children who had access to remote symptom monitoring and cognitive and behavioral skills training for pain management evidenced significantly fewer moderate to severe pain episodes over the course of the 60-day study compared to children who only reported on their symptoms daily. Furthermore, children in 
the intervention condition did not report any moderate to severe pain episodes in the latter parts of the study.

The modest, but promising, effects of Pain Buddy on pain during cancer treatment are comparable to similar mHealth pediatric pain intervention studies ${ }^{19}$. Similar to the effects of WebMap ${ }^{15}$, which also provides evidence-based pain management skills training for pediatric pain, both groups in the present study demonstrated pain reductions over the course of the study but there were no significant differences in the change of pain intensity between the attention control and intervention groups. However, our trial uniquely demonstrated that children in the intervention group reported fewer instances of moderate to severe pain over the course of the study. This implies that Pain Buddy may be particularly advantageous for assisting children who have significantly elevated levels of pain and also has implications for outcomes of pain intervention studies. Furthermore, similar interventions with mobile pain assessment and realtime pain management support, such as Pain Squad+, have demonstrated comparable adherence rates for pain assessment completion and have been shown to reduce pain for children ${ }^{14}$, however, efficacy has not yet been demonstrated in a randomized controlled trial. Thus, this pilot study of Pain Buddy offers several distinctive contributions to the pediatric mHealth literature.

More specifically, when placed in the context of existing literature, our findings suggest that it is likely that pain experienced by children during cancer treatment decreases over time perhaps regardless of the various interventions examined. This was demonstrated both in a prior examination of Pain Squad + as well as in the findings of the present study; that is, users of the previous interventions experienced significant reductions in pain severity over time. However, the prior evaluation of Pain Squad+ did not contain a comparison group and was an examination of pain over time in one group of children using the intervention. Conversely, the present 
investigation evaluated Pain Buddy using an RCT study design, which allowed for a comparison of the intervention to an attention control group. This provided an opportunity to discern whether the intervention itself had an impact on pain severity or whether pain severity simply decreases over time throughout cancer treatment and/or whether monitoring pain perhaps contributes to reductions in pain severity over time. By comparing pain monitoring (our attention control group) with monitoring, skills training, and remote symptom assessment (our intervention group), we were able to discern potential active treatment components. In fact, whereas we did not see significant group differences in pain severity over time, suggesting that either the act of monitoring influences pain severity or severity decreases as an effect of time, we did see significant impacts of the intervention on the frequency of painful episodes over time. Thus, our between-subjects comparison of the intervention and attention control groups advances our understanding of the impact of mHealth interventions for pain by suggesting that pain severity may not be the most important outcome of interest and that these interventions may be particularly efficacious for reducing frequency of pain episodes over time rather than average pain severity over time. Accordingly, this study informs important outcomes when assessing the impact of mHealth interventions for cancer-related pain by suggesting that focusing exclusively on pain severity may not be sufficient and perhaps broadening the focus to assess pain episodes as well is more indicative of the targeted impact of interventions that include both pain monitoring and skills training. Moreover, these findings indicate that Pain Buddy is a promising mHealth intervention for pain management in children undergoing cancer treatment.

\section{Limitations \& Future Directions}

Although it was expected that the intervention group would experience significantly greater reductions in pain over the course of the 60-day study compared to the attention control 
group, this was not the case. It is likely that because pain reports were skewed (the median was 0 for both groups), there may have been a floor effect that limited the potential effectiveness of the intervention. Thus, it may be important to focus on children who are actively experiencing pain with such an intervention. Furthermore, engagement with the skills training was lower than expected in this trial and may have hindered the effectiveness of the intervention. Efforts to increase engagement with mHealth interventions is vital. Finally, because this was a pilot study, the participant sample was small, which may have limited the findings.

Based upon this initial pilot, we are currently conducting a large-scale, multi-site trial of Pain Buddy with the aim of gathering effectiveness data. These early results guided several modifications to the Pain Buddy program as well as our procedures and patient population in order to increase engagement and be able to focus on a broader range of outcomes. More specifically, we gamified Pain Buddy to increase engagement, are recruiting only children who have experienced clinically significant pain in the month prior to study enrollment, and recruiting children who are within the first 16 weeks of a cancer diagnosis, a period when pain may be at its highest ${ }^{20}$. Once we complete our effectiveness trial, our goals are to examine ways in which Pain Buddy may need to be tailored to be relevant to specific populations (e.g., a wider age range, different cultural backgrounds) as well as to partner with hospitals in order to make Pain Buddy available to children undergoing cancer treatment. Pain Buddy data are clinically relevant, are designed to improve the clinical care of children undergoing cancer treatment, and could ultimately become part of a child's medical record.

Our goal is to bridge the gap in knowledge translation in the management of pain in children undergoing cancer treatment and demonstrate that the use of mHealth programs such as Pain Buddy will provide an avenue in which to accomplish this aim. Continued stakeholder 
involvement in the development of Pain Buddy, large-scale efficacy testing, and dissemination to those in need will ensure that Pain Buddy continues to have a positive impact on pediatric pain management. 


\section{Funding}

This project was funded in part by a grant from the American Cancer Society (MRSG-13-05301-PCSM; PI: MA Fortier)

\section{Conflict of Interest}

None

\section{Acknowledgements}

None 


\section{REFERENCES}

1. American Cancer Society. Cancer Facts \& Figures 2018. Atlanta, GA; 2018. https://www.cancer.org/research/cancer-facts-statistics/all-cancer-facts-figures/cancerfacts-figures-2018.html.

2. Miller E, Jacob E, Hockenberry M. Nausea, pain, fatigue, and multiple symptoms in hospitalized children with cancer. Oncol Nurs Forum 2011;38:E382-E393. doi:10.1188/11.ONF.E382-E393

3. Fortier MA, MacLaren JE, Martin SR, Perret-Karimi D, Kain ZN. Pediatric pain after ambulatory surgery: Where's the medication? Pediatrics 2009;124:e588-e595. doi:10.1542/peds.2008-3529

4. Hunter JF, Kain ZN, Fortier MA. Pain relief in the palm of your hand: Harnessing mobile health to manage pediatric pain. Pediatr Anesth 2019;29:120-124. doi:10.1111/pan.13547

5. Fortier MA, Chung WW, Martinez A, Gago-Masague S, Sender L. Pain buddy: A novel use of m-health in the management of children's cancer pain. Comput Biol Med. 2016;76:202-214. doi:10.1016/j.compbiomed.2016.07.012

6. Jacob E, Stinson J, Duran J, et al. Usability testing of a smartphone for accessing a webbased e-diary for self-monitoring of pain and symptoms in sickle cell disease. J Pediatr Hematol Oncol. 2013;34:326-335. doi:10.1097/MPH.0b013e318257a13c.Usability

7. Baggott C, Gibson F, Coll B, Kletter R, Zeltzer P, Miaskowski C. Initial evaluation of an electronic symptom diary for adolescents with cancer. J Med Internet Res. 2012;14. doi:10.2196/resprot.2175

8. Bates DW, Kuperman GJ, Wang S, et al. Ten commandments for effective clinical decision support: Making the practice of evidence-based medicine a reality. $J$ Am Med 
Informatics Assoc. 2003;10:523-530. doi:10.1197/jamia.M1370.Although

9. Mehling WE, Hamel KA, Acree M, Byl N, Hecht FM. Randomized, controlled trial of breath therapy for patients with chronic low-back pain. Altern Ther Health Med. 2005;11:44-52.

10. Kwekkeboom K, Wanta B, Bumpus M. Individual difference variables and the effects of progressive muscle relaxation and analgesic imagery interventions on cancer pain. Jounral Pain Symptom Manag. 2008;86:3279-3288. doi:10.1007/s11103-011-9767-z.Plastid

11. Fors EA, Sexton H, Götestam KG. The effect of guided imagery and amitriptyline on daily fibromyalgia pain: A prospective, randomized, controlled trial. J Psychiatr Res. 2002;36:179-187. doi:10.1016/S0022-3956(02)00003-1

12. Kleiber C, Harper D. Effects of distraction on children's pain and distress during medical procedures: A meta-analysis. Nurs Res. 1999;48:44-49. doi:10.1097/00006199199901000-00007

13. Stinson JN, Jibb LA, Nguyen C, et al. Development and testing of a multidimensional iphone pain assessment application for adolescents with cancer. $J$ Med Internet Res. 2013;15. doi:10.2196/jmir.2350

14. Palermo TM, Law EF, Fales J, Bromberg MH, Jessen-Fiddick T, Tai G. Internet-delivered cognitive-behavioral treatment for adolescents with chronic pain and their parents: A randomized controlled multicenter trial. Pain. 2017;157:174-185. doi:10.1097/j.pain.0000000000000348.

15. Jibb LA, Stevens BJ, Nathan PC, et al. Implementation and preliminary effectiveness of a real-time pain management smartphone app for adolescents with cancer: A multicenter pilot clinical study. Pediatr Blood Cancer. 2017;64:1-9. doi: 10.1002/pbc.26554 
16. Hertzog MA. Considerations in determining sample size for pilot studies. Res Nurs Health. 2008;31(2):180-191

17. Collins JJ, Devine TD, Johnson EA, Pinkerton CR, Thaler HT. The measurement of symptoms in young children with cancer: The validation of the memorial symptom assessment scale in children aged 7 - 12. J Pain Symptom Manage. 2002;23:10-16.

18. Jacob E, Mack K, Savedra M, Van Cleve L, Wilkie D. Adolescent pediatric pain tool (APPT) for multidimensional measurement of pain in children and adolescents. Pain Manag Nurs. 2014;15:694-706. doi:10.1016/j.pmn.2013.03.002.

19. Fisher E, Heathcote L, Palermo TM, Williams AC, Lau J, Eccleston C. Systematic review and meta-analysis of psychological therapies for children with chronic pain. J Pediatr Psychol. 2014; 39:763-782.

20. Van Cleve L, Bossert E, Beecroft P, Adlard K, Alvarez O, Savedra MC. The pain experience of children with leukemia during the first year of diagnosis. Nursing Research. 2004; 53:1-10 


\section{FIGURE LEGEND}

FIGURE 1. Participant flow diagram through the Pain Buddy pilot study

FIGURE 2. Average daily pain significantly declined over the course of the study for both groups. However, there were no significant differences in pain trajectory between the control and Pain Buddy groups. Error bars represent 95\% confidence intervals. 


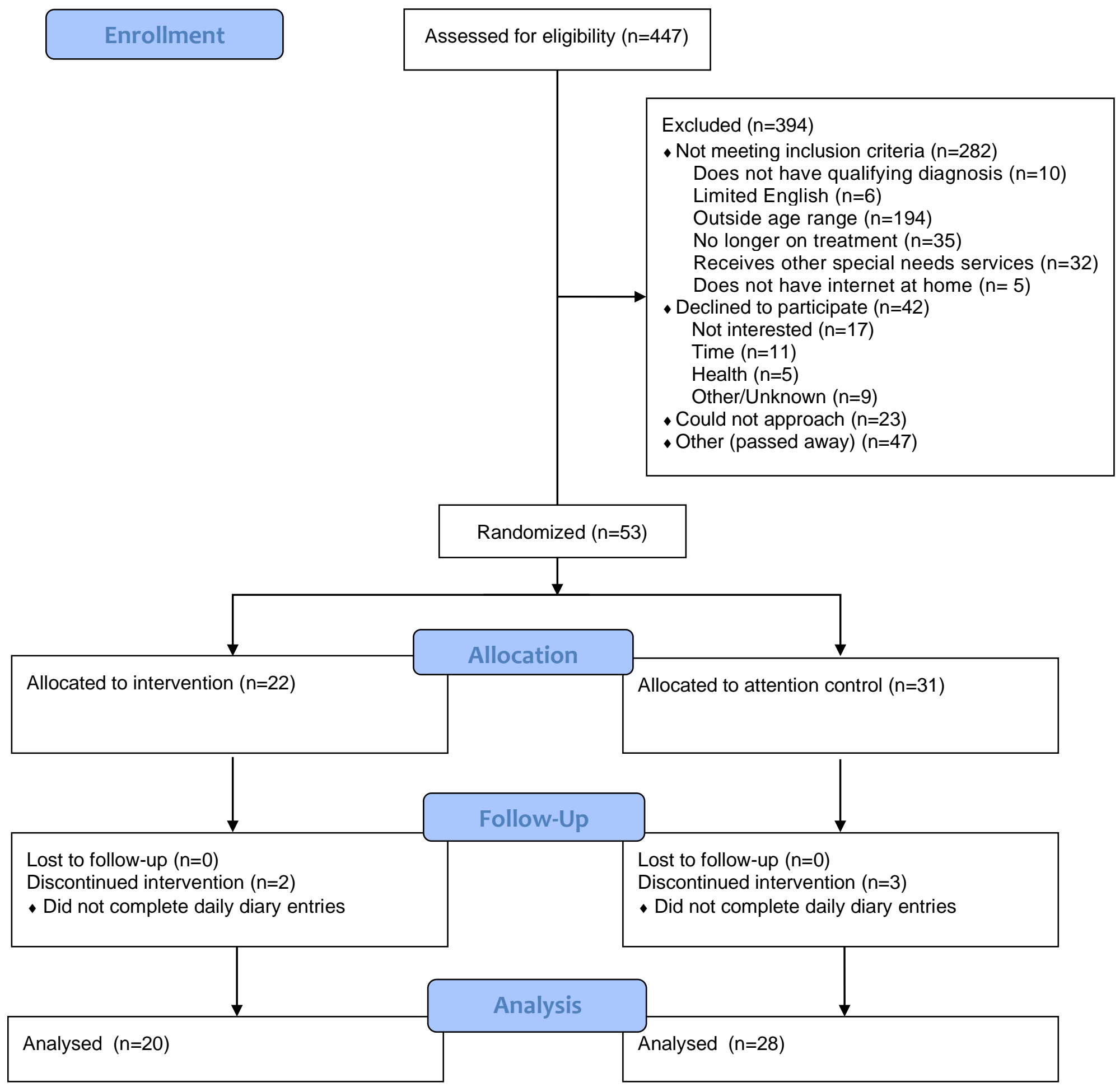




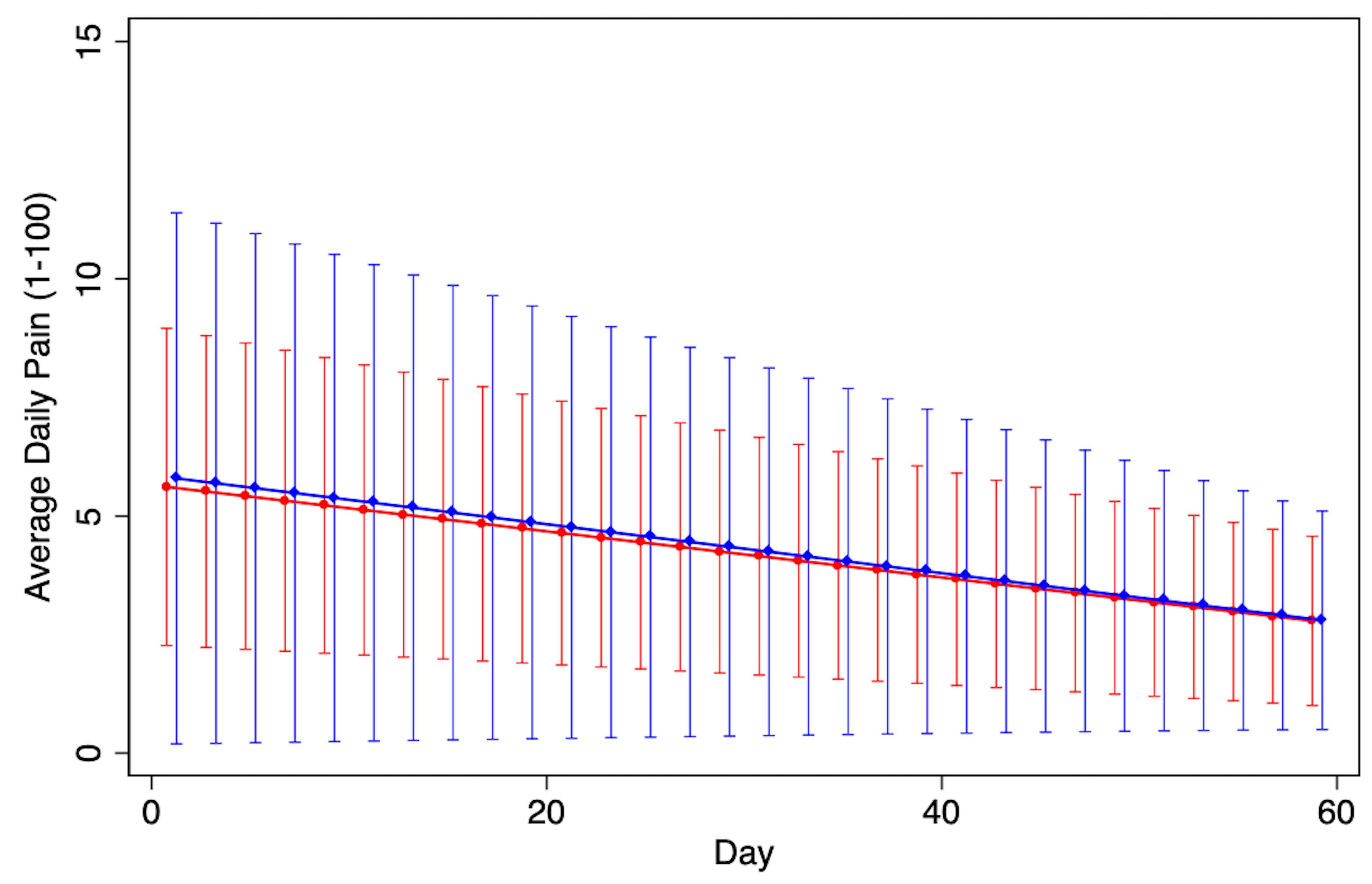


TABLE 1 Demographic characteristics did not differ by treatment condition.

\begin{tabular}{|c|c|c|c|c|}
\hline Demographics & Intervention, $n=20$ & Control, $n=28$ & $t / \chi^{2} / z$ & $p$ \\
\hline $\operatorname{Sex}^{\mathrm{a}}$ & & & 1.34 & 0.25 \\
\hline Male & $12(63.2)$ & $22(78.6)$ & & \\
\hline Female & $7(36.8)$ & $6(21.4)$ & & \\
\hline Race/ Ethnicity a & & & 7.87 & 0.34 \\
\hline Hispanic & $12(60.0)$ & $21(77.8)$ & & \\
\hline White & $5(25.0)$ & $2(7.4)$ & & \\
\hline Asian & $3(15.0)$ & $2(7.4)$ & & \\
\hline Other & $0(0.0)$ & $2(7.4)$ & & \\
\hline Primary Language at Home a & & & 1.79 & 0.78 \\
\hline English & $10(52.6)$ & $16(57.1)$ & & \\
\hline Spanish & $8(42.1)$ & $9(32.1)$ & & \\
\hline Other & $1(5.3)$ & $3(10.7)$ & & \\
\hline Diagnosis $^{\text {a }}$ & & & 3.77 & 0.44 \\
\hline Leukemias & $17(85.0)$ & $17(60.7)$ & & \\
\hline Lymphomas & $1(5.0)$ & $3(10.7)$ & & \\
\hline Sarcomas & $1(5.0)$ & $3(10.7)$ & & \\
\hline CNS Tumors & $1(5.0)$ & $3(10.7)$ & & \\
\hline Other & $0(0.0)$ & $2(7.1)$ & & \\
\hline Months Since Diagnosis ${ }^{b}$ & $19.50(26.35)$ & $19.39(20.12)$ & 0.02 & 0.99 \\
\hline $\mathrm{Age}^{\mathrm{b}}$ & $12.25(3.58)$ & $11.86(3.44)$ & 0.38 & 0.70 \\
\hline Median Family Annual Income ${ }^{c}$ & $\begin{array}{c}29,000.00 \\
(14,400.00- \\
45,000.00)\end{array}$ & $\begin{array}{c}37,500.00 \\
(20,100.00- \\
78,750.00)\end{array}$ & -1.11 & 0.27 \\
\hline
\end{tabular}

Note: Some demographic data was missing, however, percentages reported reflect the proportion of the available data (i.e. valid percent). Abbreviations: $\mathrm{CNS}=$ Central Nervous System

${ }^{\mathrm{a}} n(\%)$

${ }^{\mathrm{b}} M(S D)$

${ }^{\mathrm{c}}$ median (interquartile range) 